\begin{tabular}{|c|c|}
\hline Title & Transient A nalysis of a Stripline Having a Corner in Three-Dimensional Space \\
\hline Author(s) & Yoshida, N.; Fukai, I. \\
\hline Citation & IEEE Transactions on Microwave Theory and Techniques, 32(5), 491-498 \\
\hline Issue Date & $1984-05$ \\
\hline Doc URL & http:/hdl .handle.net/2115/5961 \\
\hline Rights & $\begin{array}{l}\text { (1984 IEEE. Personal use of this material is permitted. However, permission to reprint/republish this material for } \\
\text { advertising or promotional purposes or for creating new collective works for resale or redistribution to servers or lists, } \\
\text { or to reuse any copyrighted component of this work in other works must be obtained from the IEEE. } \\
\text { IEEE, IEEE Transactions on Microwave Theory and Techniques, 32(5), 1984, p491-498 }\end{array}$ \\
\hline Tyре & article \\
\hline File Information & ITMTT32-5.pdf \\
\hline
\end{tabular}

Instructions for use 


\title{
Transient Analysis of a Stripline Having a Corner in Three-Dimensional Space
}

\author{
NORINOBU YOSHIDA AND ICHIRO FUKAI
}

\begin{abstract}
The transient analysis of electromagnetic fields has shown its utility not only in clarifying the variation of the fields in time but also in gaining information on mechanisms by which the distributions of an electromagnetic field at the stationary state are brought about. We have recently proposed a new numerical method for the transient analysis in three-dimensional space by formulating the equivalent circuit based on Maxwell's equation by Bergeron's method. The resultant nodal equation is uniquely formulated in the equivalent circuit for both the electric field and the magnetic field. In this paper, we deal with the stripline which should be analyzed essentially in three-dimensional space because of its structure. The time variation of the electric and magnetic field of the stripline having a corner is analyzed and the remarkable changing of distribution of the field is presented as a parameter of time and of conditions imposed by the corner structure.
\end{abstract}

\section{INTRODUCTION}

$\mathrm{T}$ HE TRANSIENT ANALYSIS of electromagnetic fields not only clarifies the variation of the fields in time but also provides information on mechanisms by which the distributions of electromagnetic fields at the stationary state are brought about. We have recently proposed a new numerical method for the transient analysis in three-dimensional space [1], [2]. The method was based on the equations obtained by Bergeron [3]. The equations show the character of the propagation of electromagnetic waves in the equivalent circuit based on Maxwell's equation [4]. This method has two important advantages for the analysis. One is the formulation of the electromagnetic fields in terms of the variables in the equivalent circuits. This treatment enables us to see that the nodal equation is uniquely formulated in the equivalent circuit for both the electric field and the magnetic field because of the duality of both field components. The other advantage is the formulation by Bergeron's method with its many merits; such as the representation of the medium by the lumped elements at each node and its reactive characteristics which are represented by the trapezoidal rule of the differential equation in the time domain. This treatment is based on an iterative computation in time using only the values obtained after the previous step. Consequently, the savings in memory storage space and computer time is remarkable. The formulation of this method is fundamentally equivalent to that of the Transmission-Line Matrix (TLM), because both methods are based on the property of the traveling wave, that is formulated as the general solution of one-dimensional wave equation by d'Alembert. But the Bergeron's formulation [5], in terms of the voltage variable

Manuscript received July 20, 1983; revised November 28, 1983.

The authors are with the Department of Electrical Engineering, Faculty of Engineering, Hokkaido University, Sapporo, 060 Japan.

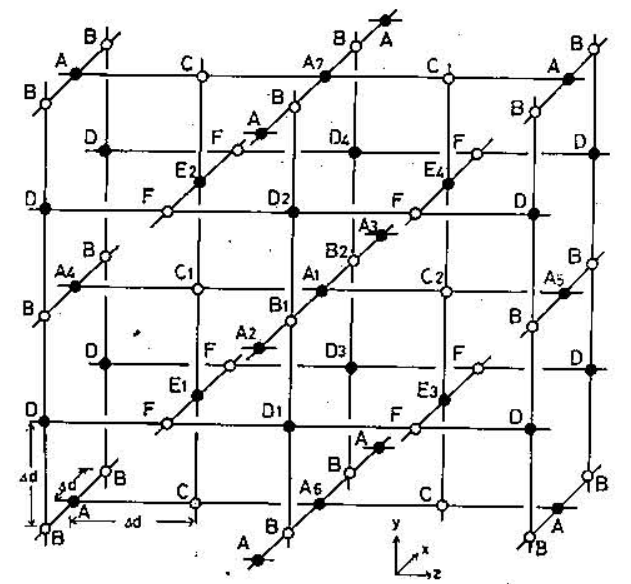

Fig. 1. Three-dimensional lattice network model of Maxwell's equations.

and current variable, presents the direct handling of the electromagnetic field variables and the characteristics of the medium instead of the division of each variable into the incident and reflective components and the composition of those in TLM method [6].

In this paper, we deal with the stripline which should be analyzed essentially in three-dimensional space because of its structure. The stripline is widely used as the transmission medium in MIC design, and its small size compared to the wavelength is the main reason for the good performance of microwave components and usually permits the treatment of circuits as a system composed of lumped elements in the analysis. But in high-frequency application, such as millimeter-wave devices, especially when using the pulse-wave technique that has progressed remarkably with the digital technique, the exact treatment of the higher components in the spectrum of the waves are indispensable, so the distributed formulation of the devices in threedimensional space is essential.

In the following sections, the fundamental formulations for the stripline by our method are briefly described, and derived parameters, such as the characteristic impedance and wavelength, are examined and compared with those obtained analytically. Lastly, the time variation of the field distribution in the stripline with a corner is shown for several conditions of the structure.

\section{Three-Dimensional NodAl Formulation of MaXWELl's EQUATION}

\section{A. Three-Dimensional Equivalent Circuit}

We now consider the formulation of nodal equations for three-dimensional analysis of the electromagnetic field in 
TABLE I

CORRESPONDENCES BETWEEN THE FIELD VARIABLES IN MaXWELl's EQuation AND THE EQUIVALENT CIRCUIT AT EACH Kind of NoDE IN THE EQuivalent Circuit

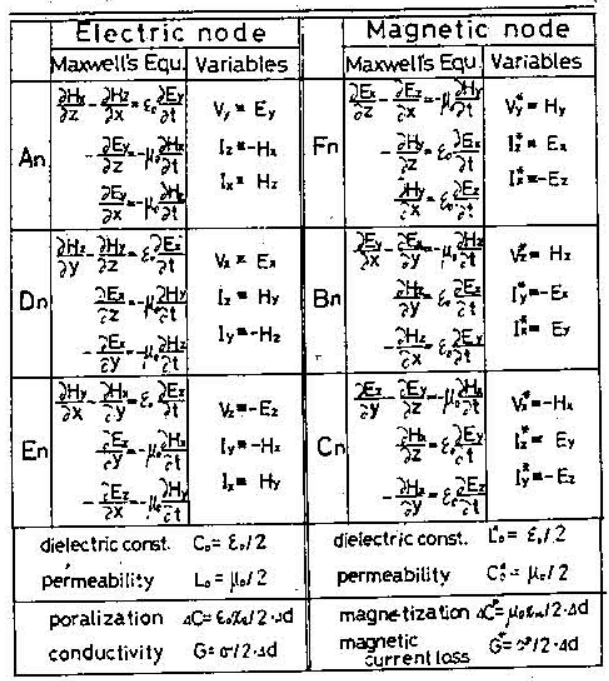

the time domain. In Fig. 1, the three-dimensional network model is shown. It is well known that this network gives a fundamental connection between the field variables in Maxwell's equation. This model is used in other methods, such as the "TLM" by P. B. Johns. In this network, each set of two-dimensional equations for the propagation of waves in each plane is related to a node and the connected lines. We interpret this network as the equivalent circuit, in which the line between nodes is a one-dimensional transmission line and the node is the point where the continuity of currents occurs. In Table I, the correspondence between the equivalent circuit variables and field quantities are shown at every kind of node of the network. The nodes are classified into two types. One is the electric node at which an electric field component is treated as a voltage variable and the other is a magnetic node at which a magnetic field component is treated as a voltage variable. The electric node corresponds to the shunt node and the magnetic node correspond to the series node in the "TLM". However, in our method the introduction of the magnetic current in the magnetic nodes results in the existence of the shunt node only in a sense of "TLM", where the continuity of current is postulated. In this paper, all variables at the magnetic nodes are characterized by the symbol " *" because of the duality of their physical meaning, as compared with their interpretation at the electric node. In Fig. 2, the fundamental connection between the nodes in the network is expressed. The correspondence of the variables is also illustrated in each node and each transmission line. The direction of the Poynting vector, which is decided by the set of an electric and a magnetic field component supposed in each one-dimensional transmission line, is also shown. Each of the supposed directions of the Poynting vector coincides with that of the currents in both nodes of the transmission line, so the currents are defined as the usual conduction currents in the electrical circuit. The gyrator is

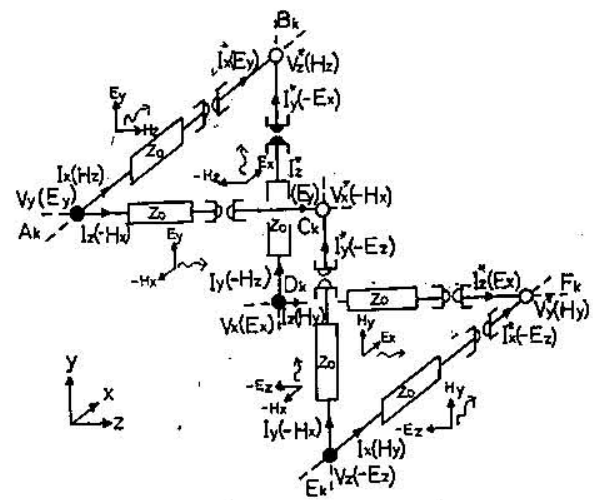

(a)

$$
\begin{aligned}
& v \stackrel{I}{\rightarrow} 3 \epsilon^{I^{*}} v^{*} \quad v-\stackrel{I}{\longrightarrow} \rightarrow E^{I^{*}} v^{*} \\
& \left(\begin{array}{l}
v \\
1
\end{array}\right)=\left(\begin{array}{ll}
0 & 1 \\
1 & 0
\end{array}\right)\left(\begin{array}{l}
v^{*} \\
l^{*}
\end{array}\right) \quad\left(\begin{array}{l}
v \\
I
\end{array}\right)=\left(\begin{array}{cc}
0 & -1 \\
-1 & 0
\end{array}\right)\left(\begin{array}{l}
v^{*} \\
l^{*}
\end{array}\right)
\end{aligned}
$$

(1)

(b)

Fig. 2. (a) Fundamental connection of the node in the network and detailed expression of the variables in the equivalent circuit. The direction of the Poynting vector is shown by the symbol " $\rightarrow$ " at each" transmission line. (b) Definition of gyrator in (a), (1) positive gyrator, and (2) negative gyrator.

inserted in series with each magnetic node to show the duality of the physical meaning of the circuit variables of both nodes of each transmission line. At $D_{n}$ nodes, negative gyrators are inserted, since the corresponding circuit variables and electromagnetic variables have a polarity oppo:site to that at the nodes $B_{n}$. We interpret this negative gyrator as a circuit representation of the self-consistence of the Maxwell's equations, and the node to be inserted is determined by the correspondence of circuit variables and electromagnetic variables.

\section{B. Bergeron's Method}

Next, we formulate the propagation characteristics of a one-dimensional transmission line by Bergeron's method. In Fig. 3(b), showing a section of lossless line, the propagation characteristics of waves in the time domain are given by the one-dimensional wave-equation

$$
\begin{aligned}
& v(k, t)+z \cdot i(k, t)=v(k-1, t-\Delta t)+z \cdot i(k-1, t-\Delta t) \\
& v(k-1, t)-z \cdot i(k-1, t)=v(k, t-\Delta t)-z \cdot i(k, t-\Delta t)
\end{aligned}
$$

where the parameter $k$ denotes the node numbers and $z$ is the characteristic impedance of the line, $t$ is time and $\Delta t$ is the transit time between two adjacent nodes, which also becomes the fundamental time step in the numerical com? putation. Each lumped element to be connected with the line at nodes is characterized as follows: the conductance $G$ is expressed in terms of its branch voltage $v_{g}$ and current $i_{g}$ by

$$
v_{g}(k, t)=G(k) i_{g}(k, t) .
$$




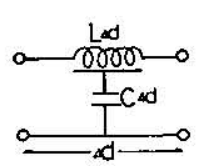

(a)

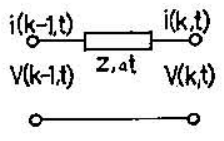

(b)
Fig. 3. Typical equivalent circuit (a) of the one-dimensional transmission line and its description (b) by means of Bergeron's method.

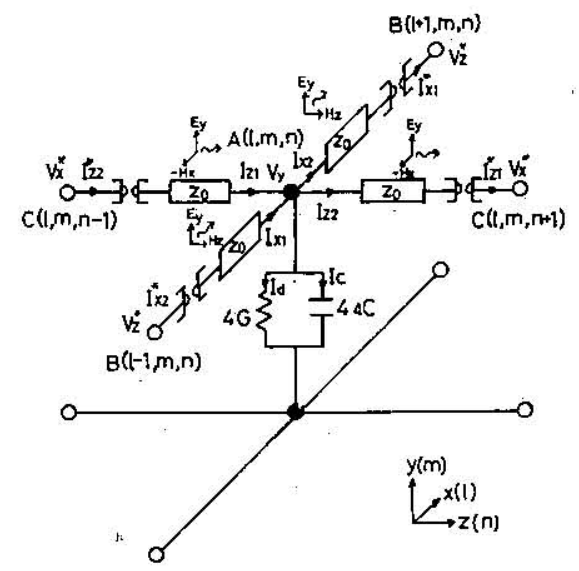

Fig. 4. Equivalent circuit at each $A_{n}$ node in the dielectric medium. Dielectric loss is expressed by the parallel conductance.

In this equation, the conductance $G$ is a function of the parameter $k$ at each node. The capacitance $C(k)$ is defined through the trapezoidal rule and is given by

$$
v_{c}(k, t)-R_{c} i_{c}(k, t)=v_{c}(k, t-\Delta t)+R_{c} i_{c}(k, t-\Delta t)
$$

where

$$
R_{c}=\frac{\Delta t}{2 C(k)} .
$$

In this equation, $\left(v_{c}, i_{c}\right)$ is a pair of branch voltages and currents of the capacitance. A comparison of both sides in (1a), (1b), and (3a) shows that all values calculated at time $t$ are only a function of ones at the previous time $t-\Delta t$. The nodal equation for each node at time $t$ is independent of the values of the adjacent node at time $t$. The time responses are thus iteratively computed at each time, $t$ from values of the circuit values at every node obtained at the previous steps.

\section{Three-Dimensional Nodal Equations}

For the three-dimensional network, the characteristics of the transmission line are formulated by Bergeron's method. In Fig. 4, the example of the equivalent circuit is shown at the node $A_{n}$ where the electric field $E_{y}$ is supposed to be a voltage variable and the magnetic fields $-H_{x}$ and $H_{z}$ are supposed to be the current components in the directions $z$ and $x$, respectively. Application of (1a) and (1b) to each line connected to the node yields the following equation:

$$
\begin{aligned}
& V_{y}(l, m, n, t)+z_{0} I_{z 1}(l, m, n, t) \\
& \quad=I_{z 2}^{*}(l, m, n-1, t-\Delta t)+z_{0} V_{x}^{*}(l, m, n-1, t-\Delta t)
\end{aligned}
$$

$$
\begin{aligned}
& V_{y}(l, m, n, t)-z_{0} I_{z 2}(l, m, n, t) \\
& \quad=I_{z 1}^{*}(l, m ; n+1, t-\Delta t)-z_{0} V_{x}^{*}(l, m, n+1, t-\Delta t)
\end{aligned}
$$

$$
\begin{aligned}
& V_{y}(l, m, n, t)+z_{0} I_{x 1}(l, m, n, t) \\
& \quad=I_{x 2}^{*}(l-1, m, n, t-\Delta t)+z_{0} V_{z}^{*}(l-1, m, n, t-\Delta t)
\end{aligned}
$$

$$
\begin{aligned}
& V_{y}(l, m, n, t)-z_{0} I_{x 2}(l, m, n, t) \\
& \quad=I_{x 1}^{*}(l+1, m, n, t-\Delta t)-z_{0} V_{z}^{*}(l+1, m, n, t-\Delta t) .
\end{aligned}
$$

The parameters $l, m$, and $n$ denote the described position numbers of $x, y$, and $z$ directions, respectively. Then (2) is written with the notation of this case as follows:

$$
V_{y}(l, m, n, t)=4 G(l, m, n) I_{d}(l, m, n, t) \text {. }
$$

Equations (3a) and (3b) are again written as follows:

$$
\begin{aligned}
& V_{y}(l, m, n, t)-R_{c} I_{c}(l, m, n, t) \\
& \quad=V_{y}(l, m, n, t-\Delta t)+R_{c} I_{c}(l, m, n, t-\Delta t)
\end{aligned}
$$

where

$$
R_{c}=\frac{\Delta t}{8 \Delta C(l, m, n)} .
$$

The conductance $G$ and capacitance $\Delta C$ are listed in Table I and are shown to correspond to the conductive loss and the electrical displacement of the dielectric medium, respectively. The conductance $G$ also corresponds to the equivalent dielectric loss in the medium. These quantities are considered to be a function of the position variables $l$, $m$, and $n$. The continuity of the current at node $\dot{A}(l, m, n)$ is given by

$$
I_{z 1}-I_{z 2}+I_{x 1}-I_{x 2}-I_{d}-I_{c}=0 .
$$

Substituting (4a)-(4d), (5), and (6a) into (7), the unified nodal equation in a dielectric medium with conductive or equivalent dielectric loss is given by

$$
V_{y}(1, m, n, t)=\frac{R_{c} \cdot\left(\Psi_{1}^{*}+\Psi_{2}^{*}+\Psi_{3}^{*}+\Psi_{4}^{*}\right)+z_{0} \Psi_{c}}{z_{0}+R_{c} \cdot\left(4+z_{0} \cdot 4 G(l, m, n)\right)}
$$

where $\Psi_{1}^{*}, \Psi_{2}^{*}, \Psi_{3}^{*}$, and $\Psi_{4}^{*}$ correspond to the right-hand sides of (4a)-(4d), respectively, and $\Psi_{c}$ is equal to the right side of (6a). Equation (8) is iteratively evaluated at every $A_{n}$ node, and the time response of the field in the overall region is analyzed by the same procedure at other kinds of nodes. Each component of the currents at the time $t$ is evaluated by substituting $V_{y}(t)$ in (8) by $V_{y}(t)$ in the left sides of (4a)-(4d), (5), and (6a). The other variables at other nodes also are obtained in the same manner. For example, the voltage variable $V_{y}^{*}(t)$ in the magnetic nodes $F_{n}$ is given as follows:

$$
V_{y}^{*}\left(l^{\prime}, m^{\prime}, n^{\prime}, t\right)=\frac{R_{c}^{*} \cdot\left(\Psi_{1}+\Psi_{2}+\Psi_{3}+\Psi_{4}\right)+z_{0}^{*} \Psi_{c}^{*}}{z_{0}^{*}+R_{c}^{*} \cdot\left(4+z_{0}^{*} \cdot 4 G^{*}\left(l^{\prime}, m^{\prime}, n^{\prime}\right)\right)}
$$




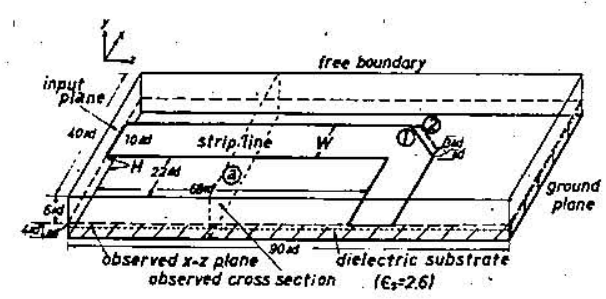

Fig. 5. Geometry of a stripline with a $90^{\circ}$ bend.

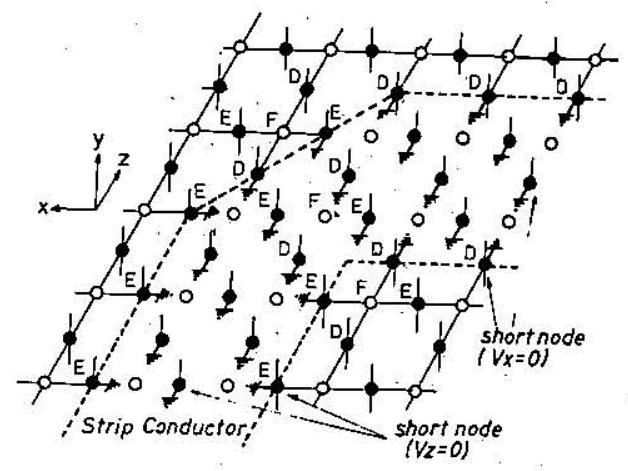

Fig. 6. Equivalent circuit of the surface of the stripline with infinite conductivities.

where

$$
R_{c}^{*}=\frac{\Delta t}{8 \Delta C^{*}\left(l^{\prime}, m^{\prime}, n^{\prime}\right)} .
$$

In such magnetic nodes, the conductance $G^{*}$ and the capacitance $\Delta C^{*}$ have duality with those in electric nodes. These then correspond to losses of the magnetic current and the magnetization. But (8) and (9) have the same form and calculations are the same as for the electric nodes. These characteristics occur as a consequence of the application of the duality between the electric field and the magnetic field in Maxwell's equations. Thus, the introduction of magnetic currents is an important concept in this method and constitutes the difference between this method and the "TLM" [7]. Both methods are fundamentally based on the d'Alembert's general solution for one-dimensional wave equation, but the use of both voltage and current variables in this method enables us to express the characteristic equation of the medium by lumped circuit element instead of the artificial stub in "TLM". This formulation is extended to more complex characteristics such as dispersive, resonance, and anisotropic media [8].

\section{NuMERICAL RESUltS AND Discussion}

The transient analysis for the stripline with a corner has been performed by the method described in the preceding section. In Fig. 5, the model of the stripline with the corner is shown. In this figure, $\Delta d$ is the interval between adjacent nodes in the equivalent circuit. In order to describe this model by the "Nodal Equation", three different conditions are introduced, namely, the boundary condition at the strip conductor, the boundary condition at the free boundary, which is supposed to be the surface of the analyzed region in air and dielectric medium, and the condition of the

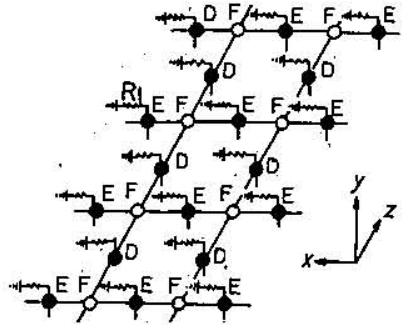

(a)

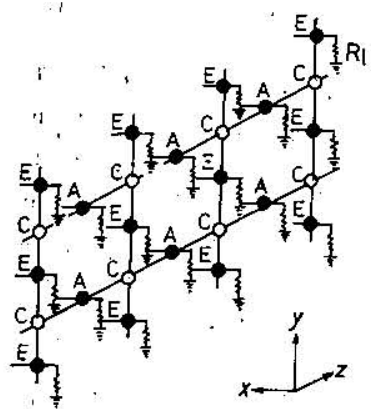

(b)

Fig. 7. Equivalent circuit of the free boundary. (a) The $x z$-plane of the top of the analyzed region. (b) The $y z$-plane of the side of the region, where $R_{1}$ is the characteristic impedance of the free space.

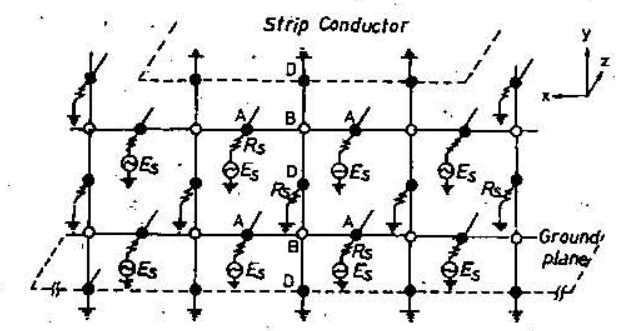

Fig. 8. Equivalent circuit of the input condition, where $R_{s}$ is the characteristic impedance of the stripline, and $E_{s}$ is the voltage source, in this analysis, of the sinusoidal wave expression as $E_{s}=E_{o} \sin (2 \pi / T) n \Delta t$, ( $E_{o}$ : Amplitude, $T$ : Period of the sinusoidal wave, $n$ : Number of iteration).

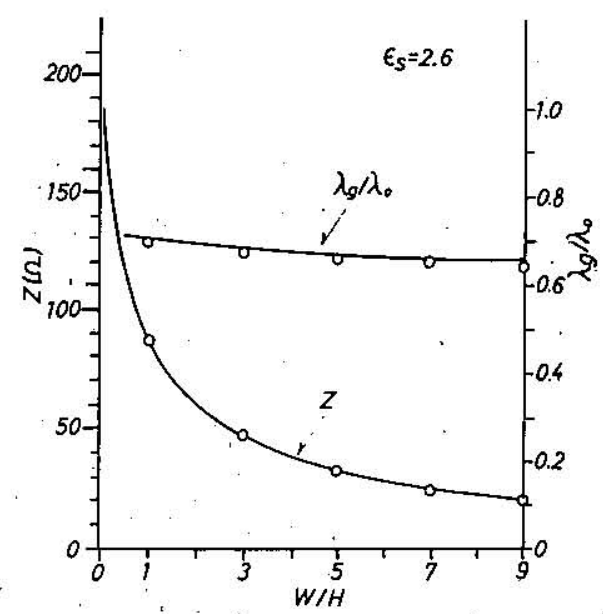

Fig. 9. Characteristic impedance and wavelength as a function $W / H$ of a straight stripline. - --- result computed by our method, analytical results by $\mathrm{E}$. Yamashita and $\mathrm{R}$. Mittra.

dielectric. Firstly, the boundary condition of the conductor is described. The conductor is supposed to have infinite conductivity, so the tangential component of electric fields on the surface of the conductor should be zero. This 


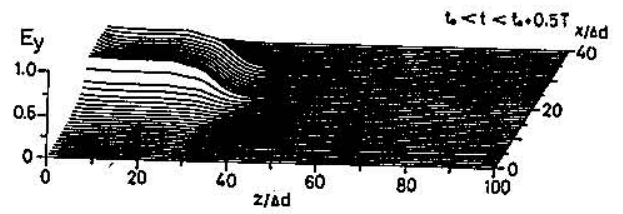

(a)

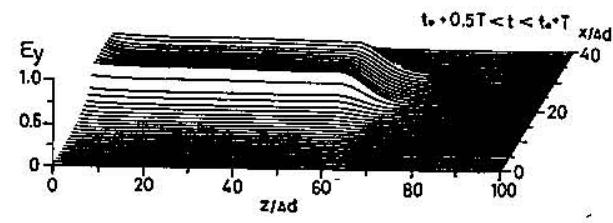

(b)

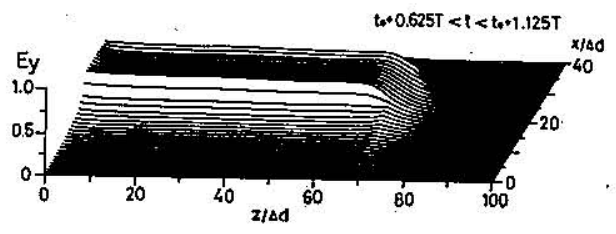

(c)

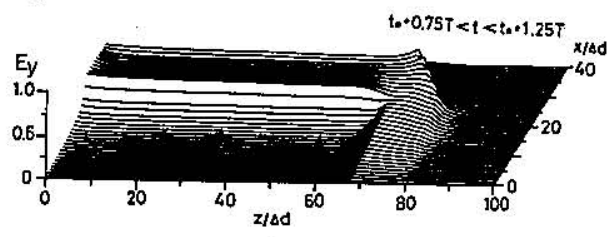

(d)

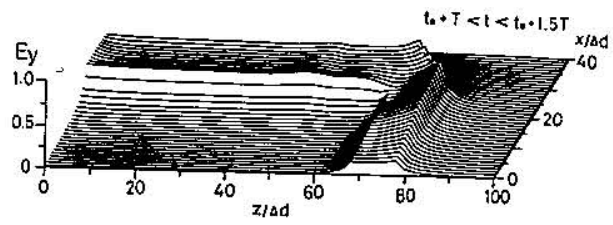

(e)

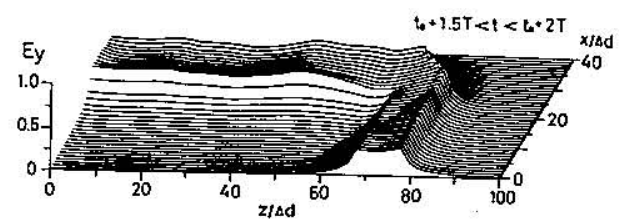

(f)

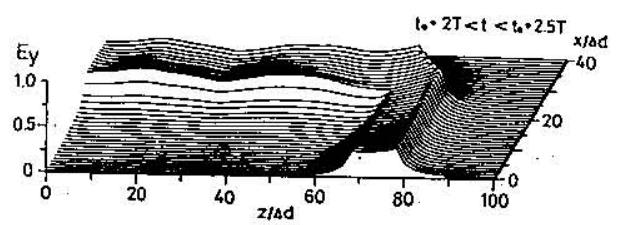

(g)

Fig. 10. Time variation of the distribution of the electrical field $E_{y}$ on the observed $x z$-plane shown in Fig. 5 and in the case of the corner pattern (1), where $t_{o}$ is the initial time at which the input wave is applied to the input plane. $T$ is the period of the applied sinusoidal wave.

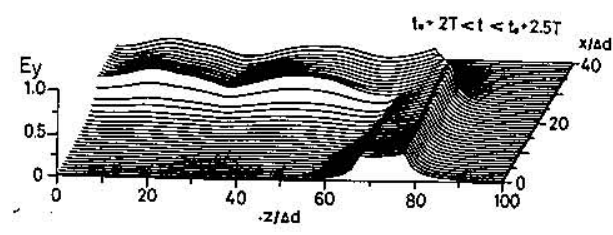

Fig. 11. Time variation of the distribution of the electrical field $E_{y}$ in the case of the corner pattern (2) in Fig. 5.

condition is realized by short-circuiting the appropriate electric node, in which the tangential electric field at the surface is the voltage variable, and by open-circuiting the appropriate magnetic node, in which the tangential component of the electric field is the current variable, that is, it is considered as a magnetic current. These situations are shown in Fig. 6. In the equivalent circuit, the plane in which the strip conductor is positioned is arbitrarily defined. In this analysis, the plane is situated at the plane containing $D_{n}, F_{n}$, and $E_{n}$ nodes. Thus, the $D_{n}$ and $E_{n}$ nodes are short-circuited, because the electric fields $E_{x}$ and $E_{z}$ are to be zero at both surfaces of the conductor, and $F_{n}$ nodes are neglected because all the field components $E_{x}$,
$E_{z}$, and $H_{y}$ are equal to zero on the surface. Next, the free boundary condition is -expressed as a nonreflective termination, at which the load resistance, equal to the characteristic impedance of the free space, approximates the matching condition. The equivalent circuit of this condition is shown in Fig. 7, in which (a) shows the upper plane of the analyzed region and (b) shows the side plane. Finally, the characteristics of the dielectric are expressed in terms of the equivalent parallel circuit composed of the capacitance and the conductance at the electric node in the dielectric medium, as shown in Fig. 4. The physical meaning of the lumped element is shown in Table I and the formulation of this elements is expressed in (5) and (6). At the node situated on the dielectric-air interface, the value of the capacitance is assigned to be one half of that in the inner node. In this analysis, losses in the dielectric medium have been neglected.

Using this model of the stripline, the transient analysis of the stripline with a corner has been performed. The input condition is assumed as follows in the equivalent circuit: A sinusoidal voltage wave is applied through the source resistance at the $A_{n}$ nodes under the stripline on the 


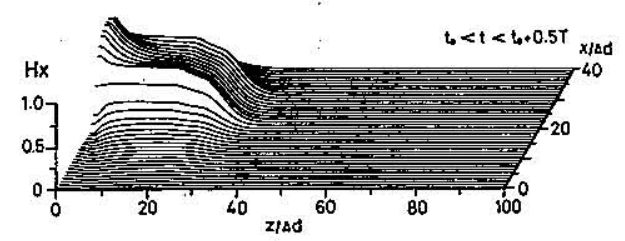

(a)

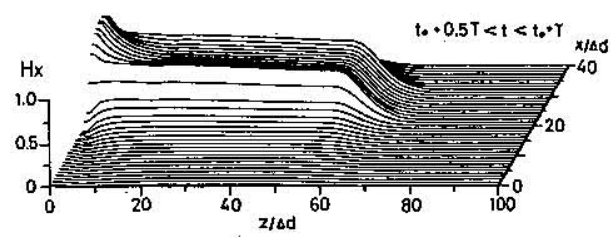

(b)

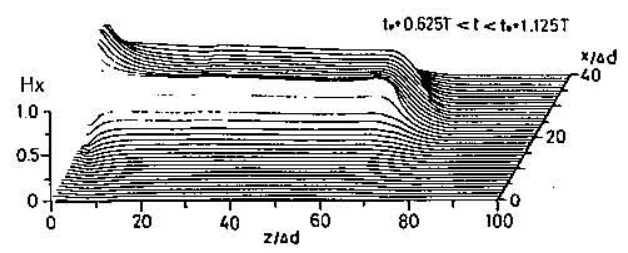

(c)

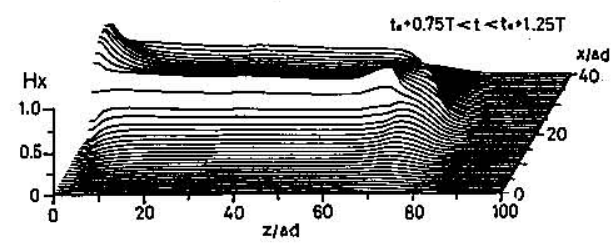

(d)

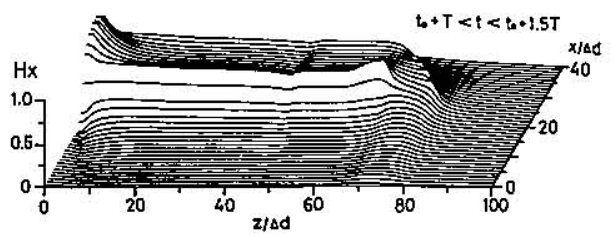

(e)

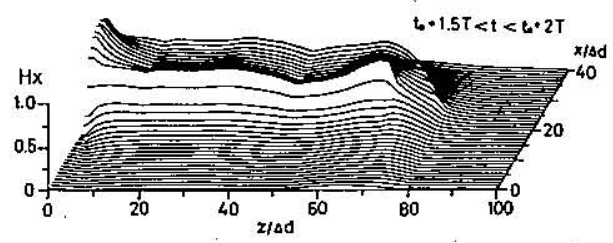

(f)

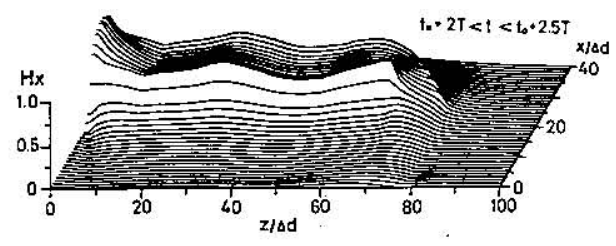

(g)

Fig. 12. Time variation of the distribution of the magnetic field $H_{x}$ on the observed $x z$-plane in Fig. 5 for the same case as Fig. 10.

input plane. The source impedance is equal to the characteristic impedance of the dominant mode in the dielectric medium. This input condition approximates the excitation of the TEM-wave. The other $A_{n}$ nodes and $D_{n}$ nodes in the input plane are terminated by a matching impedance. The configuration of the input plane is shown in Fig. 8. The shape of the input wave is expressed as a pulse train with a spacing $\Delta t$ in the time domain. In this numerical evaluation, the interval $\Delta d$ between adjacent nodes in the equivalent is circuit is chosen, for example, to be $0.005 \mathrm{~cm}$. Then, the time interval $\Delta t$ becomes $8.333 \times 10^{-5} \mathrm{~ns}$. The period of the applied sinusoidal wave is $213 \Delta t$ in this analysis, so its frequency is about $56 \mathrm{GHz}$. These values of $\Delta d$ and $\Delta t$ are sufficiently small so that the resolution of the spatial and time function is satisfactory. In the numerical computation, all parameters in space and time are normalized to $\Delta d$ and $\Delta t$, respectively.

In Fig. 9, the numerical results for the characteristic impedance and wavelength are plotted as a function of $W / H$, and compared with analytically obtained curves.
This figure shows that the numerical results are in close agreement with the analytical ones. In the following figures, the spatial distribution of the field at each time is obtained by taking the maximum values in the half period of the applied wave because of computations on the time axis. The $x z$-plane on which we observe the field is that of $\Delta d$ beneath the upper strip conductor as shown in Fig. 5 as "observed plane". The initial point of the time axis is assumed to be the point at which the incident wave is applied at the input plane.

Fig. 10 shows the time variations of the electrical field $E_{y}$ in the case of the corner cut pattern (1) given in Fig. 5 . It is observed that the propagating wave curves. the corner smoothly and the VSWR at the incident side is small. This result clearly shows that the cutting pattern of the corner is suitable. However, Fig. 11 shows a comparatively large VSWR for the other cutting pattern (2) shown in Fig. 5 These results show that the cutting pattern of the corner influences the propagation characteristics considerably Figs. 12 and 13 present the time variations of the magnetic 


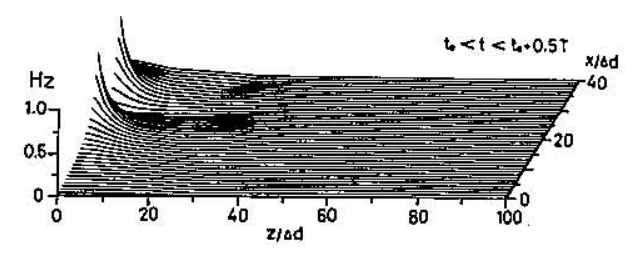

(a)

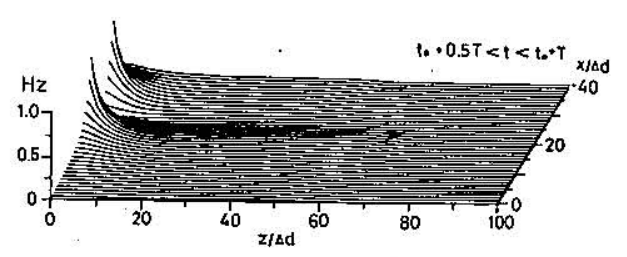

(b)

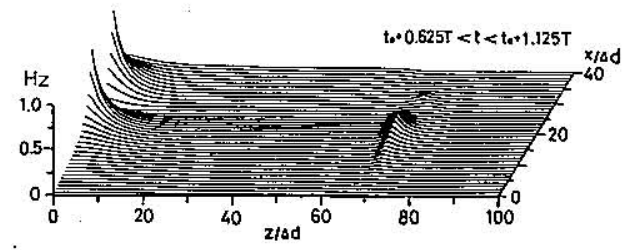

(c)

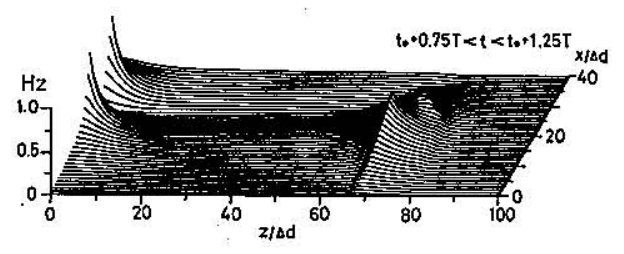

(d)

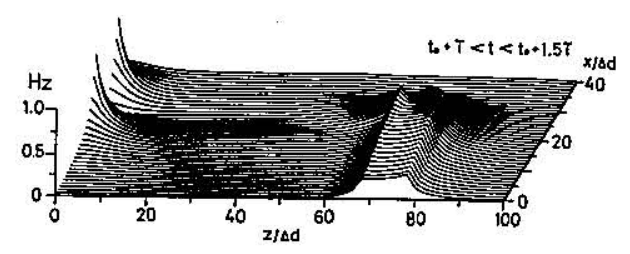

(e)

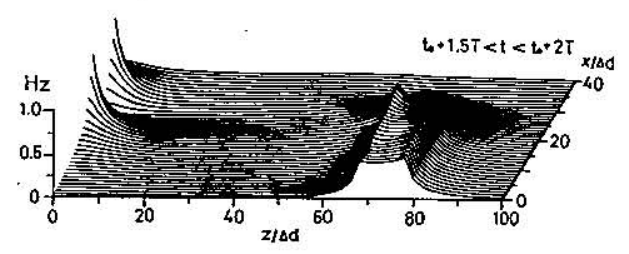

(f)

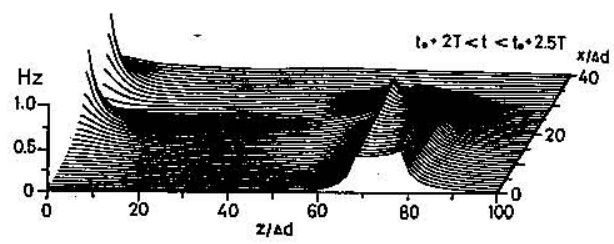

(g)

Fig. 13. Time variation of the distribution of the magnetic field $H_{z}$ in the same case as Fig. 10 .

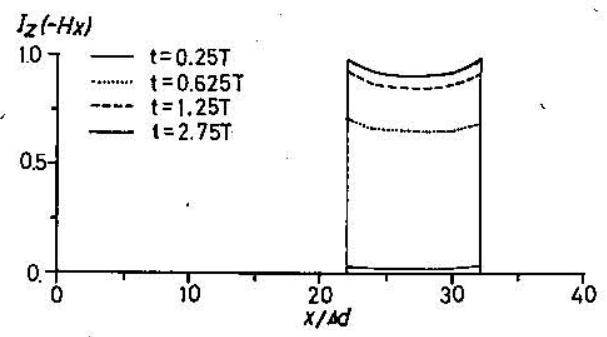

Fig. 14. Time variation of the magnetic field $H_{x}$ at the cross section (a) in Fig. 5. This magnetic component corresponds to the current in the z-direction.

field $H_{x}$ and $H_{z}$, respectively, for the case of Fig. 10. Each figure clearly shows that the conversion of the magnetic component $H_{x}$ to the component $H_{z}$ occurs at the corner. These magnetic components are the dominant terms of the Poynting vector in each longitudinal section of the stripline. Both figures show that near the input port, both magnetic components $H_{x}$ and $H_{z}$ are generated by diffraction of the input wave because of the plane-wave ap- proximation of the input conditions. Fig. 14 shows the time variation of the magnetic field $H_{x}$ at the cross section (a) shown in Fig. 5, where the steady-state distribution is established and the edge effect is evient.

The size of the program used is about 4.5 MB, and the computed time for the transient analysis from $t=0$ to $t=2.5 \mathrm{~T}$ is about $80 \mathrm{~s}$.

\section{CONCLUSION}

The present study verifies that our method is appropriate for the time-domain analysis of the stripline in threedimensional space. The obtained results demonstrate the propagation of the wave through a $90^{\circ}$ bend and show how the direction of the magnetic field is changed by the corner contour. We are now studying in more detail the time variations of fields in striplines as a function of many other parameters and examine the relation between the propagation characteristics for transient distributions and stationary continuous waves and the pulsed wave. These results will be reported in later papers. 
The present method can also be applied to other threedimensional problems by using all merits of the method [9]-[11].

\section{REFERENCES}

[1] N. Yoshida, I. Fukai, and J. Fukuoka, "Transient analysis of two-dimensional Maxwell's equations by Bergeron's method," Trans. IECE Japan, vol. J62-B, pp. 511-518, Jun. 1979.

[2] __. "Transient analysis of three-dimensional electromagnetic fields by nodal equations," Trans. IECE Japan, vol. J63-B, pp. 876-883, Sept. 1980.

[3] G. Metzger and J-P. Vabre, Transmission Lines with Pulse Excitation. New York: Academic Press, 1969, pp. 65-96.

[4] S. Akhtarzad and P. B. Johns, "Solution of Maxwell's equations in three space dimensions and time by the t.1.m. method of numerical analysis," Proc. IEE, vol. 122, pp. 1344-1348, Dec. 1975.

[5] H. W. Dommel and W. S. Meyer, "Computation of electromagnetic transients," Proc. IEEE,-vol. 62; pp. 983-993, July 1974.

[6] S. Akhtarzad and P. B. Johns, "Generalized elements for TLM method of numerical analysis," Proc. IEE, vol.122, pp. 1349--1352, Dec. 1975.

[7] P. B. Johns and M. O'Brien, "Use of the transmission-line modelling (t.1.m.) method to solve non-linear lumped networks," Radio Electron. Eng., vol. 50, pp. 59-70, Jan./Feb. 1980.

[8] N. Yoshida, I. Fukai, and J. Fukuoka, "Application of Bergeron's method to anisotropic media," Trans. IECE Japan, vol. J64-B, pp. 1242-1249, Nov. 1981.

[9] ___ "Adaptation of Bergeron's method to complicated boundary problems," Trans. IECE Japan, vol E64, pp. 455-462, July 1981.

[10] , "Transient analysis of waveguide having $H$-comer," Trans, $\overline{I E C E}$ Japan, vol. E65, pp. 125-126, Feb. 1982.

[11] __ "Transient analysis of waveguide having tuning window," Trans. IECE Japan, vol. E66, pp. 161-162, Feb. 1983.

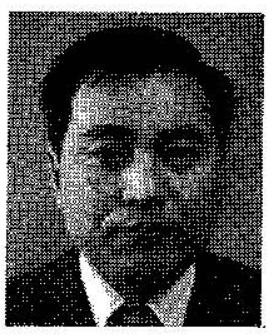

Norinobu Yoshida was born in Hokkaido Japan, on May 27, 1942. He received the B.S. and M.S. degrees in electronics engineering from Hokkaido University, Sapporo, Japan, in 1965 and 1967, respectively, and received the D.E. degree in electrical engineering from the same university in 1982.

He joined Nippon Electric Co., Ltd., Tokyo, in 1967, and engaged in CAD at the Integrated Circuit Division. He became a Research Assistant in 1969 in the Department of Electrical Engineering in the Faculty of Engineering of Hokkaido University. Since 1983, he has been a lecturer of the same department. He presently is engaged in research of numerical methods for transient analysis of electromagnetic fields.

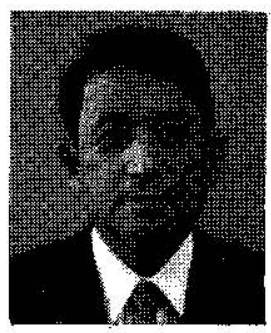

Ichiro Fukai was born in Hokkaido, Japan, on August 21, 1930. He receive the B.S., M.S., and D.E. degrees in electrical engineering from Hokkaido University, Sapporo, Japan, in 1953, 1956 , and 1976, respectively.

In 1956, he joined the Defense Agency Technical Research and Development Institute. He became a Research Assistant in 1959 in the Faculty of Engineering of Hokkaido University, Assistant Professor of the Technical Teacher's Training Institute in 1961, Assistant Professor in 1968, and Professor in 1977 in the Department of Electrical Engineering of the Faculty of Engineering of the university. 